Journal of Economics and Behavioral Studies

Vol. 5, No. 3, pp. 157-163, Mar 2013 (ISSN: 2220-6140)

\title{
The Role of Model Complexity and the Performance of the Selection Criteria in Asymmetric Price Transmission Models
}

\author{
${ }^{1}$ Henry de-Graft Acquah*, ${ }^{2}$ Patrick Ofosuhene \\ ${ }^{1}$ University of Cape Coast, Ghana \\ ${ }^{2}$ North Carolina A \& T State University, Ghana \\ *henrydegraftacquah@yahoo.com
}

\begin{abstract}
The role of model complexity in asymmetric price transmission model selection is not well understood. In order to appreciate the role of model complexity in model selection performance, this study fits alternative asymmetric price transmission models that differ in complexity to simulated data and evaluates the ability of the model selection method to recover the true model. The results of Monte Carlo experimentation suggest that in general BIC, CAIC and DIC were superior to AIC when the true data generating process was the Manning Error Correction model (MECM). However, AIC was more successful when the true model was the Complex Error Correction Model (CECM). The tendency of the complex model (CECM) to over fit the relatively simpler true asymmetric data generating process (MECM) is minimized in larger samples. The research findings demonstrate the role of model complexity in asymmetric price transmission model comparison and selection.
\end{abstract}

Keywords: Asymmetry, Monte Carlo, Complexity, Information Criteria, Recovery Rate

\section{Introduction}

Dynamics in price transmission has attracted considerable research interest among agricultural economists. Of particular interest is the issue of asymmetric price transmission. This recognition has led to the formulation of variants of econometric models to describe the phenomena. The various model specifications include Houck (1977), Manning (1991) and Von Cramon-Taubadel and Loy (1996). Fundamentally, these competing specifications differ in model complexity. The problem of selecting a model that best captures the underlying asymmetric price transmission process arises as more competing models become available. This issue of model selection has been investigated using information criteria. For example, Acquah (2010), Monte Carlo comparison of model selection methods finds that though the selection criteria points to the true model, their performance improved with increase in sample size and decrease in error variance. However, Acquah (2010) did not consider the influence of model complexity in asymmetric price transmission model selection analysis. Though the influence of model complexity in the selection of an asymmetric price relationship is not well understood, the issue has not received considerable attention. For instance, the issue of whether a complex asymmetric price transmission model will provide a better fit when the true data generating process is a simplier model remains unaddressed. Myung (2000) in a study of mathematical models of cognitive psychology asserts that the ability of the model selection methods to select the true model out of a set of competing models is influenced by model complexity and sample size. In effect he notes that the model selection methods are sensitive to model complexity. However, very little work has been done in the asymmetric price transmission modeling framework to ascertain the role of model complexity in asymmetric price transmission model selection. The thrust of this paper is to support the claim that, the model selection methods are sensitive to model complexity and demonstrate the role of complexity in asymmetric price transmission model selection. Specifically, asymmetric price transmission models which differ in complexity are used to investigate how model selection criteria are sensitive to complex models with the aid of simulated data.

\section{Literature Review}

Alternative models have been developed to study the asymmetric price transmission (APT) process. Some of the commonly used asymmetric price transmission model includes those developed by Houck (1977), 
Manning (1991), and Von Cramon-Taubadel and Loy (1996). Houck (1977) applies a simple but rigorous model to study irreversible supply functions. In the Houck's approach, asymmetries are specified to affect the direct impact of price increases and decreases and do not take into account adjustments to the equilibrium level. Manning (1991) extended the Houck's approach to include equilibrium relationship. The contribution by Manning (1991) emerged as he studied the relationship between retail price, excise duties and crude oil prices in the UK using monthly data over a period of 1973-1988. Retail prices were expressed as a function of crude oil prices and taxes. He suggested that in the presence of cointegrated variables, the residuals from the regression are stationary and can be introduced as an additional regressor. While Manning's methodology test for asymmetries in the direct impact of a price increase and decrease, Von Cramon-Taubadel and Loy (1996) developed a method that focused on asymmetries in the adjustment to the equilibrium. The authors studied the relationship between retail and wholesale prices of pork in the German market, using weekly data over the period January 1990-October 1993. Capps and Sherwell (2007) notes that though the alternative methods or models are continually used in analyzing price dynamics and testing for asymmetric adjustments to derive policy conclusions, they remain incompatible with one another and may result to differences in inference and conclusions. This necessitates the need to apply an objective set of criterion to choose amongst the different models given a particular data. In support, Manera and Frey (2007) assert that no attempt has been made to address the issue of which of the various asymmetric price transmission models is most reliable or fits a given data set better, despite the numerous empirical research undertaken. In effect, there has been very little basis for choosing between the different methods. However, model selection methods such as Akaike and Bayesian Information Criteria (Akaike, 1973 and Schwarz, 1978) and their analytical extensions provide a basis for choosing between asymmetric price transmission models. Acquah (2010) notes that the model selection methods do points to the true data generating process in asymmetric price transmission analysis. However, Acquah (2010) fails to examine the effect of complexity on model selection performance in asymmetric price transmission analysis.

\section{Methodology}

The methodology describes the asymmetric price transmission models employed in the study, the model selection methods and the simulated data generating process of the study. Asymmetric price transmission models such as the Manning's model, Von Cramon-Taubadel and Loy Model and the Houck's model are emphasized. The model selection technique emphasizes the Akaike and Bayesian Information Criteria and their analytical extensions.

Asymmetric Price Transmission Models (APT): The Manning (1991) Error Correction Model (ECM) data generating process (DGP) can be specified as follows:

$\Delta P_{A, t}=\beta_{1}^{+} \Delta P_{B, t}^{+}+\beta_{1}^{-} \Delta P_{B, t}^{-}+\beta_{2} E C T_{t-1}+\varepsilon \quad \varepsilon \square N\left(0, \sigma^{2}\right)$

$\Delta P_{B, t}^{+}$and $\Delta P_{B, t}^{-}$are the positive and negative changes in $P_{B, t}$. Asymmetric behavior is then measured by allowing the speed of adjustment to differ for the positive and negative components of $P_{B, t}$. Symmetry in equation (1) is detected using an F-test of the null hypothesis that the coefficients ( $\beta_{1}^{+}$and $\beta_{1}^{-}$) are identical (i.e. $H_{0}: \beta_{1}^{+}=\beta_{1}^{-}$). Noticeably, Manning (1991) does not segment the Error Correction Term into positive and negative components.

Von Cramon-Taubadel and Loy (1996) propose a complex asymmetric Error Correction Model data generating process which can be specified as follows:

$$
\Delta P_{A, t}=\beta_{1}^{+} \Delta P_{B, t}^{+}+\beta_{1}^{-} \Delta P_{B, t}^{-}+\beta_{2}^{+} E C T^{+}{ }_{t-1}+\beta_{2}{ }^{-} E C T^{-}{ }_{t-1}+\varepsilon \quad \varepsilon \square N\left(0, \sigma^{2}\right)
$$


where $\Delta P_{B, t}^{+}$and $\Delta P_{B, t}^{-}$are the positive and negative changes in $P_{B, t}$. The Error Correction Term (ECT) is partitioned into positive and negative deviations resulting in the asymmetric error correction model defined in equation (2). Where,

$$
\begin{aligned}
& E C T^{+}{ }_{t-1}=E C T_{t-1} \text { if } E C T_{t-1}>0 \text { and } 0 \text { otherwise, and } \\
& E C T_{t-1}^{-}=E C T_{t-1} \text { if } E C T_{t-1}<0 \text { and } 0 \text { otherwise. }
\end{aligned}
$$

Asymmetric behavior is then measured by allowing the differing speed of adjustments for the positive and negative components of the Error Correction Term (ECT) since the equilibrium relationship captured by the ECT was implicitly symmetric, Cook, Holly and Turner (1999).

Noticeably, a formal test of the asymmetry hypothesis using equation (2) is: $\boldsymbol{H}_{\mathrm{o}}: \boldsymbol{\beta}_{\mathbf{1}}{ }^{+}=\boldsymbol{\beta}_{\mathbf{1}}{ }^{-}$and $\beta_{2}^{+}=\beta_{2}^{-}$. In effect, asymmetric behavior is assessed by a joint F-test.

The Houck's data generating process can be written as follows:

$$
\Delta \boldsymbol{P}_{A, t}=\boldsymbol{\beta}_{1}^{+} \Delta \boldsymbol{P}_{B, t}^{+}+\boldsymbol{\beta}_{1}^{-} \Delta \boldsymbol{P}_{B, t}^{-}+\boldsymbol{\nu}_{t} \quad \boldsymbol{v}_{t} \square N\left(0, \sigma_{v}^{2}\right)
$$

where $\Delta P_{B, t}^{+}$and $\Delta P_{B, t}^{-}$are the positive and negative changes in $P_{B, t}$. Symmetry is tested by determining whether the coefficients $\left(\beta_{1}^{+}\right.$and $\beta_{1}^{-}$) are identical (i.e. $H_{0}: \beta_{1}^{+}=\beta_{1}^{-}$).

Model Selection Criteria: In asymmetric price transmission applications, multiple models are often considered. The aim of asymmetric price transmission is to choose one of the models based on information criteria. Information criteria provide an attractive basis for model selection. Various information criteria have been developed. Generally, these model selection methods adjust for a variation in the number of parameters among models, essentially penalizing models with additional parameters. They include the Akaike information criterion (AIC; Akaike, 1973); Consistent Akaike information criterion (CAIC; Bozdogan, 1987); the Bayesian information criterion (BIC; Schwarz, 1978); and the Draper's Information Criteria (DIC; Draper, 1995). The model selection methods are specified as follows:

$$
\begin{aligned}
& A I C=-2 \log (L)+2 p \\
& C A I C=-2 \log (L)+p[(\log n)+1] \\
& B I C=-2 \log (L)+p \log (n) \\
& D I C=-2 \log (L)+p \log (n / 2 \pi)
\end{aligned}
$$

Where $L$ refers to the likelihood under the fitted model, $p$ is the number of parameters in the model and $n$ is the sample size. Models that minimize the AIC, BIC, CAIC and DIC are selected. AIC differs from CAIC, BIC and DIC in the second term which now takes into consideration sample size $n$. Thus CAIC, BIC and DIC allows for asymptotic consistency.

Data Generating Process: The study uses artificial data. This study draws from the experimental design of Holly et al (2003) and Acquah (2010) and specifies the Manning ECM (MECM) and complex Von CramonTaubadel and Loy ECM (CECM) data generating process as follows and with an error variance of 1 .

$$
\Delta P_{A, t}=0.25 \Delta P_{B, t}^{+}+0.75 \Delta P_{B, t}^{-}-0.50 E C T_{t-1}+\varepsilon
$$




$$
\Delta P_{A, t}=0.95 \Delta P_{B, t}^{+}+0.20 \Delta P_{B, t}^{-}-0.25 E C T_{t-1}^{+}-0.75 E C T_{t-1}^{-}+\varepsilon
$$

The variables in the model remain as defined previously under the asymmetric price transmission models.

\section{Results and Discussion}

In order to simulate the effects of sample size and model complexity on model selection, this study considers three sample sizes ranging from small to large corresponding to 50,150 and 500. Using an error size of 1, the data was generated from the Manning Error Correction Model (MECM) specified in equation (8). The results of the Monte Carlo simulations comparing the performance of the model selection methods are displayed in Table 1. It is essential to note that all recovery rates are derived using 1000 Monte Carlo simulations. In effect, the amount of samples in which each model fits better than the other competing models is measured out of the 1000 samples and expressed as a percentage. In this context, the values derived from each model by selection methods are derived as the arithmetic mean based on 1000 samples. For the purpose of brevity, the Manning asymmetric error correction model, the complex asymmetric error correction model and the Houck's model are denoted by MECM, CECM and HKD respectively.

Table 1: Relative Performance of Model Selection Methods across Sample Size

\begin{tabular}{|c|c|c|c|c|c|}
\hline \multirow{2}{*}{$\begin{array}{l}\text { Sample Size } \\
50\end{array}$} & \multicolumn{4}{|c|}{ Model Fitted } & \multirow[b]{2}{*}{ MECM(DGP) } \\
\hline & & \multicolumn{2}{|c|}{ CECM } & HKD & \\
\hline & Meth & & & & \\
\hline & AIC & \multicolumn{2}{|c|}{ 118(18.6\%) } & 125 ( $5.8 \%)$ & $117(75.6 \%)$ \\
\hline & BIC & \multicolumn{2}{|c|}{126 (5.9\%) } & 130 (15.1\%) & 124 (79\%) \\
\hline & CAIC & \multicolumn{2}{|c|}{$129(3.2 \%)$} & $130(20.9 \%)$ & $125(75.9 \%)$ \\
\hline & DIC & \multicolumn{2}{|c|}{$129(4.6 \%)$} & 131(14.1\%) & $126(81.3 \%)$ \\
\hline \multirow[t]{6}{*}{150} & & \multicolumn{2}{|c|}{ CECM } & HKD & MECM(DGP) \\
\hline & Meth & & & & \\
\hline & AIC & 402 & $(15 \%)$ & $431(0 \%)$ & 401 (85\%) \\
\hline & BIC & 416 & $(2.7 \%)$ & 440 (0 \%) & $412 \quad(97.3 \%)$ \\
\hline & CAIC & 417 & $(1.6 \%)$ & $439(0.2 \%)$ & 413 (98.2\%) \\
\hline & DIC & 419 & (2\%) & $441(0.1 \%)$ & 414 (97.9\%) \\
\hline \multirow[t]{6}{*}{500} & & \multicolumn{2}{|c|}{ CECM } & HKD & MECM(DGP) \\
\hline & Meth & & & & \\
\hline & AIC & 1396 & $(14.9 \%)$ & $1503(0 \%)$ & 1395 (85.1\%) \\
\hline & BIC & 1417 & $(0.9 \%)$ & $1515(0 \%)$ & $1411(99.1 \%)$ \\
\hline & CAIC & 1416 & $(0.5 \%)$ & $1513(0 \%)$ & $1410(99.5 \%)$ \\
\hline & DIC & 1419 & $(0.7 \%)$ & $1517(0 \%)$ & $1413(99.3 \%)$ \\
\hline
\end{tabular}

Based on 1000 Replications

Generally, inspection of the recovery rates for the different model selection criteria illustrates the extent to which the true model (MECM) is recovered by each selection criteria across the different sample sizes. In the small sample size of 50, at least 75.6 percent of the true model was recovered across the model selection methods in the top part of Table 1. At a sample size of 500, at least 85.1 percent of the true model was recovered by the model selection methods. The results also indicates that though the data generating process was the Manning error correction model, the model selection methods demonstrated a tendency to select the complex model even in large samples as indicated in Table 1. This may be partly due to its flexibility. For example, in large samples, AIC, CAIC, BIC and DIC demonstrated a tendency to select the complex model with recovery rates of 0.5 to 14.9 whilst the relatively simple Houck's model was not selected. However, the over fitting effects of the comparatively complex model (i.e. CECM) was greater in small samples but became less dramatic as sample size increases from 50 to 500. Similarly, in an application of model selection methods in cognitive psychology, Myung (2000) notes that the tendency of relatively complex models to over fit a simple true data generating process completely disappears in larger samples. An inference drawn here is that the 
tendency of relatively complex models to over fit a simple true asymmetric data generating process will be minimized in larger samples.

In comparison with the small sample recovery rates, model recovery of the true model improved significantly when the sample size was large. Despite differences in performance among the model selection criteria, trends holding across the different criteria were evident in the simulation results. In effect, the performance of the model selection methods to select the true model (i.e. recovery rates of MECM) generally increased with increases in sample size from 50 to 500. However, some distinct patterns can also be identified in Table 1. First, the model selection methods which takes into consideration sample size (CAIC, BIC, and DIC) performed similarly to one another with their recovery rates varying strongly as a function of sample size. Second, AIC did not make substantial gains in model recovery as sample size increased from small to large. At large sample size, AIC exhibited a stronger propensity to select complex models. This tendency is observed in the bottom part of Table 1 above where CECM is selected with a recovery rate of 14.9 percent whilst the CAIC, BIC, DIC selected the most complex model with a range of (0.5-0.9 percent). These observed patterns in the current study are consistent with previous studies on model selection. For example, Ichikawa (1988)'s simulation results in a factor analysis indicated that the ability of AIC to select a true model rapidly increased with sample size but at larger sample sizes it continued to exhibit a slight tendency to select complex models. Similarly, in a review of existing work on factor analysis, Markon and Krueger (2004) notes that AIC performs relatively well in small samples but is inconsistent and does not improve in performance in large samples whilst BIC in contrast appears to perform relatively poorly in small samples, but is consistent and improves in performance with sample size. Fishler et al. (2002) also investigated the performance of BIC in a factor analysis and their results suggest that BIC performs poorly at small sample sizes, but improves with increasing sample size to eventually choose the correct model with perfect probability. Third, the CAIC, BIC and DIC outperformed AIC across the various sample sizes given the Manning error correction model data generating process. In order to simulate the effects of sample size and model complexity on model selection, this study employed three sample sizes ranging from small to large corresponding to 50, 150 and 500. Using an error size of 1, data is generated from the Complex Asymmetric Error Correction Model (CECM) specified in equation (9). The results of the Monte Carlo simulations comparing the performance of the model selection methods are displayed below in Table 2 .

Table 2: Performance of Selection Methods across Sample Size

\begin{tabular}{|c|c|c|c|c|}
\hline Sample Size & & Model Fitt & & \\
\hline \multirow[t]{6}{*}{50} & & CECM (DGP) & HKD & MECM \\
\hline & Methods & & & \\
\hline & AIC & 118 (42.2\%) & 126 (4.7\%) & 119 (53.1\%) \\
\hline & BIC & $126(25.5 \%)$ & 131 (14.3\%) & $125(60.2 \%)$ \\
\hline & CAIC & $129(17 \%)$ & $131(20.7 \%)$ & $127(62.3 \%)$ \\
\hline & DIC & 129 (21.8\%) & 132 (16.8\%) & 127 (61.4\%) \\
\hline \multirow[t]{6}{*}{150} & & CECM (DGP) & HKD & MECM \\
\hline & Methods & & & \\
\hline & AIC & 402 (78.8\%) & $435(0 \%)$ & 406 (21.2\%) \\
\hline & BIC & 416 (53.1\%) & $444 \quad(0 \%)$ & 418 (46.9\%) \\
\hline & CAIC & 417 (44.4\%) & 443 (0 \%) & 418 (55.6\%) \\
\hline & DIC & 419 (48.3\%) & $445(0 \%)$ & $420(51.7 \%)$ \\
\hline \multirow[t]{6}{*}{500} & & CECM (DGP) & HKD & MECM \\
\hline & Methods & & & \\
\hline & AIC & 1396 (99.8\%) & 1517 (0\%) & $1412(0.2 \%)$ \\
\hline & BIC & $1417(95.7 \%)$ & 1529 (0\%) & $1429(4.3 \%)$ \\
\hline & CAIC & $1416(93.7 \%)$ & 1527 (0\%) & $1428(6.3 \%)$ \\
\hline & DIC & 1419 (94.8\%) & $1531(0 \%)$ & $1431 \quad 5.2 \%)$ \\
\hline
\end{tabular}

Based on 1000 Replications 
Generally, the performance of the different model selection criteria as the sample size increases are similar to those observed when the data was simulated from the Manning asymmetric ECM. The ability of the model selection methods to recover the true model (DGP) generally increased with sample size as shown in Table 2 . A comparison of the performance patterns of the model selection criteria illustrates that when the true model is complex, AIC persistently outperforms CAIC, BIC and DIC across moderate and large sample sizes. It is important to note that this is not the case when the true model was the Manning asymmetric ECM. Using a small sample of 50, the top part of Table 2 indicates that AIC recovers 42.3 percent of the true data generating process whilst CAIC, BIC, and DIC recovered between $17 \%$ to $25.5 \%$ percent of the true model. In large samples of 500, AIC achieve recovery of 99.8 percent whilst CAIC, BIC and DIC recovered between 93.7 to 95.7 percent when the true data generating process is complex. These results indicate that AIC persistently outperforms the other model selection methods when the true data generating process is complex. This is consistent with previous studies. For instance, Lin and Dayton (1997) find that AIC was superior to BIC when the true model was relatively complex (i.e. complexity is based on the number of parameters). Improved performance of the model selection methods when the true model is complex suggest that model complexity influence the performance of the model selection methods. An essential point is that comparatively, AIC performed better when the true asymmetric data generating is relatively complex (CECM) and the sample size is large than when the true data generating process is the Manning error correction model (MECM) and the sample size is large. This is noted when the recovery rates of Table 1 are compared with Table 2 under sample size of 500. For instance, under a sample size of 500, AIC achieved 99.8 percent recovery of the true model when the data generating process is complex but achieves 85.1 percent recovery when the true model is the Manning asymmetric error correction model (MECM). In summary, the model selection methods performed reasonably well in identifying the true model, though their ability to recover the true asymmetric data generating process increases with increase in sample size as illustrated in Tables 1 and 2. Intuitively, the results points to the fact that the sample sizes are important in the selection of the true asymmetric data generating process during price transmission analysis. Generally, larger sample sizes might improve the ability of the model selection methods to make correct inferences about asymmetric price transmission models. Another factor that may influence the performance of the model selection methods is model complexity or the number of asymmetric adjustment parameters. In large samples, AIC outperforms, CAIC, BIC and DIC when the true asymmetric data generating process is the complex error correction model. Improved model recovery rates of AIC when the true model is complex suggest that model complexity influence the performance of the model selection methods.

\section{Conclusion}

The fundamental assertion of this paper is that the performance of the model selection methods may be sensitive to model complexity in asymmetric price transmission modeling context. This study therefore examined the role of model complexity in model selection performance by fitting models that differ in complexity to simulated asymmetric data and evaluated the ability of the model selection method to recover the true model. The Monte Carlo experimentation results indicated that in general BIC, CAIC and DIC were superior to AIC when the true data generating process was the Manning error correction model, whereas AIC was more successful when the true model was the complex error correction model. This result suggests that AIC has a tendency to select more complex asymmetric price transmission models. Furthermore, the tendency of the complex (CECM) model to over fit the relatively simpler true asymmetric data generating process (MECM) is minimized in larger samples.

\section{Reference}

Acquah, H. D. (2010). Comparison of Akaike Information Criterion and Consistent Akaike Information Criterion for Model Selection in Asymmetric Price Transmission Studies. Asian-African Journal of Economics and Econometrics, 9(1), 49-56.

Akaike, H. (1973). Information Theory and an Extension of the Maximum Likelihood Principle. B. N. Petrov and F. Csaki (eds.) 2 ${ }^{\text {nd }}$ International Symposium on Information Theory: 267-81. Budapest: Akademiai Kiado.

Bozdogan, H. (1987). Model Selection and Akaike's Information Criterion (AIC): The General Theory and Its Analytical Extensions. Psychometrika, 52 (3), 345-370. 
Capps, 0. \& Sherwell, P. (2007). Alternative Approaches in Detecting Asymmetry in Farm-Retail Prices Transmission of Fluid Milk. Journal of Agribusiness, 23(3), 313-331.

Cook, S., Holly, S. \& Turner, P. (1999). The Power of Tests for Non-Linearity: The Case of Granger-Lee Asymmetry. Economics Letters, 62, 155-159.

Draper, D. (1995). Assessment and Propagation of Model Uncertainty. Journal of Royal Statistical Society. Series B (Methodological), 57(1) 45-97.

Fishler, E., Grosmann, M. \& Messer, H. (2002). Detection of signals by information theoretic criteria: general asymptotic performance analysis. IEEE Trans. Signal Process, 50(5) 1027-1036.

Holly, S., Turner P. \& Weeks, M. (2003). Asymmetric Adjustment and Bias in Estimation of an Equilibrium Relationship from a Co-integrating Regression. Computational Economics, 21, 195-202

Houck, J. P. (1977). An Approach to Specifying and Estimating Nonreversible Functions. American Journal of Agricultural Economics, 59, 570 - 572.

Ichikawa, M. (1988). Empirical Assessments of AIC Procedure for Model Selection in Factor Analysis. Behaviormetrika, 24, 33-40.

Lin, T. H. \& Dayton, C. M. (1997). Model selection Information Criteria for Non-Nested Latent Class Models. Journal of Educatational and Behavioral Statistics, 22(3) 249-264.

Manera, M. \& Frey, G. (2007). Econometric Models of Asymmetric Price Transmission. Journal of Economic Surveys, 67, 349- 415.

Manning, D. N. (1991). Petrol Prices, Oil Price Rises and Oil Price Falls: Some Evidence for the UK since 1972. Applied Economics, 23, 1535 - 1541.

Markon, K. E. \& Krueger, R. F. (2004). An Empirical Comparison of Information- Theoretic Selection Criteria for Multivariate Behavior Genetic Models. Behavior Genetics, 34(6), 593- 609.

Myung, J. I. (2000). The Importance of Complexity in Model Selection. Journal of Mathematical Psychology, 44, 190-204.

Schwarz, G. (1978). Estimating the Dimension of a Model. Annals of Statistics, 6, 461- 464.

Von-Cramon-Taubadel, S. \& Loy, J. P. (1996). Price Asymmetry in the international Wheat Market: Comment. Canadian Journal of Agricultural Economics, 44, 311-317. 